\title{
Telehealth and food insecurity screenings: challenges and lessons learned
}

\author{
Christina Bernhardt ${ }^{1}$, Christian King ${ }^{2}$ \\ ${ }^{1}$ Georgia Health Policy Center, Georgia State University, Atlanta, GA, USA; ${ }^{2}$ School of Global Health Management and Informatics, University of \\ Central Florida, Orlando, FL, USA \\ Contributions: (I) Conception and design: Both authors; (II) Administrative support: None; (III) Provision of study materials or patients: None; (IV) \\ Collection and assembly of data: None; (V) Data analysis and interpretation: None; (VI) Manuscript writing: Both authors; (VII) Final approval of \\ manuscript: Both authors. \\ Correspondence to: Christian King, PhD. University of Central Florida, DPAC 402G, 528 W Livingston St, Orlando, FL 32801, USA. Email: \\ christian.king@ucf.edu.
}

\begin{abstract}
Food insecurity remains a persistent problem in the United States and affected 35.2 million Americans in 2019. In the wake of COVID-19, food insecurity has increased in many communities. Given that food insecurity exacerbates poor health or health conditions, screening of food insecurity within medical settings is frequently identified within the literature as an important first step in effectively addressing this social concern and improving the health outcomes of patients. However, health care providers often do not screen for food insecurity for a variety of reasons. In this article review, we discuss the challenges associated with incorporating food insecurity screenings within the medical model and how the COVID-19 pandemic has exacerbated these challenges. Specifically, the COVID-19 pandemic has substantially increased the delivery of health care services via telehealth, making screening for food insecurity even more difficult via remote videoconferencing. We examine the strengths and weaknesses of telehealth and their implications for food insecurity screenings. We discuss how these implications might inform future research regarding the use of telehealth as a means of screening patients for social determinants of health in the COVID-19 era. Given that the use of telehealth is not expected to back to pre-pandemic levels, it is important to understand how to best screen for social determinants of health via videoconferencing.
\end{abstract}

Keywords: Food insecurity; provider communication; social determinants of health; patient comfort

Received: 10 August 2021; Accepted: 02 November 2021; Published: 20 January 2022.

doi: $10.21037 /$ mhealth-21-31

View this article at: https://dx.doi.org/10.21037/mhealth-21-31

\section{Introduction}

Food insecurity, the inability to access enough food for an active and healthy life (1), remains a persistent problem in the United States. In 2019, the United States Department of Agriculture (USDA) estimated that about 10.9 percent (35.2 million) of Americans experienced food insecurity at some time during 2019 (2), down from a peak of 16.6 percent (50.2 million) in 2009 during the Great Recession. The current COVID-19 pandemic has disrupted economic activity and left a substantial number of workers without employment, creating economic hardships for individuals and families across the world. Feeding America estimated that about 45 million people were food insecure in 2020, and the organization projects that about 42 million people (about 1 out of 8 Americans) will experience food insecurity in 2021 (3).

Households that are at higher risk of experiencing food insecurity include households with children; households with children under age 6 ; households with children headed by a single parent; low-income households; and households of racial minorities (2). Studies on food insecurity have documented a myriad of negative health consequences for individuals of all ages from children to elderly (4). For example, food insecurity substantially increases the risk of 
diabetes (5). Food insecure individuals are also more likely to have emergency room visits and hospital admissions (6), and food insecure adults are more likely to underuse or skip their medications due to cost $(7,8)$. As a result of these negative health consequences, the annual health care expenditures for a food insecure person are $\$ 6,071$ compared to $\$ 4,208$ for a food secure person (6).

Because of these health implications, screening of food insecurity within medical settings is frequently identified within the literature as an important first step in effectively addressing this social concern (9-11). Regular screening for food insecurity can benefit patients by increasing awareness of vital community resources, ensuring prompt referrals to resources, and decreasing the stigma surrounding food insecurity by normalizing conversations about challenges with obtaining food and other essentials that contribute to a healthy lifestyle (11). Screening patients for food insecurity in a medical setting could also help healthcare providers determine whether this underlying condition needs to be addressed before patients can be provided with an adequate health treatment.

This article presents an overview of the landscape of screening for food insecurity within healthcare settings and discusses the challenges of conducting such screenings within the medical model. Additionally, the potential implications that COVID-19, and telehealth, specifically, may have on ensuring that food insecurity screenings are performed in outpatient healthcare settings are presented. The article concludes by examining some strengths and weaknesses of telehealth that may impact provider efforts to screen patients for food insecurity and other types of social determinants of health (SDOH). We conclude with a brief discussion on possible future areas of research concerning telemedicine and food insecurity screening practices.

\section{Screening for food insecurity in healthcare settings}

The US Household Food Security Survey Module (FSSM), highly regarded for its demonstrated reliability and validity, was developed by the USDA and is an 18-item instrument that assesses an individual's risk of food insecurity (12). However, it is often unfeasible to administer this tool in healthcare settings because of the time necessary to complete the tool, which can overburden providers and patients (12). As a result, other tools derived from the FSSM attempt to alleviate the burden of administering and answering the survey for both providers and patients. For example, the
Hunger Vital Sign is a promising alternative screening option, and the condensed screening asks patients to answer the following questions: (I) How often within the past 12 months "we worried whether our food would run before we got money to buy more," and (II) The food we bought just didn't last and we didn't have money to get more (12). A patient answering "yes" to one or both questions of the tool indicate food insecurity (12). This tool has demonstrated to be comparable to the original 18-item FSSM, with researchers finding the sensitivity of the condensed Hunger Vital Sign 2-question survey to persist across multiple populations (12). Further, the brevity of the Hunger Vital Sign questionnaire makes it an ideal tool for implementation within healthcare settings, including hospitals, insurance organizations, and outpatient services (12).

Regardless of the tools available to assist with screenings, and increasing recommendations to implement screenings within medical appointments, screenings are not consistently performed by providers for a multitude of reasons (9). Previous literature notes patient and provider comfort in discussing food insecurity may influence screening practices (9). Providers who are not used or trained to screen for food insecurity might feel discomfort doing so and patients might feel uncomfortable or stigmatized from disclosing that they may be experiencing financial hardships. Patients may fear being judged or fear they may be negatively affected from disclosing financial struggle, such as having child protective services notified due to an inability to provide their children nutritious foods consistently (13). Creating a safe environment for patients, facilitated through patient-provider trust and effective communication, could help patients feel more comfortable discussing sensitive topics with healthcare providers. For example, in one qualitative study conducted in a pediatric clinic, parents noted when healthcare providers explained that the motivation behind screening for food insecurity was founded in ensuring optimal health for their children, and was not meant as a punitive measure, it helped to ease their fear and discomfort with such conversations (13). Good communication and engagement between patients and providers could help ease patient discomfort and, in turn, help providers feel more comfortable broaching the topic with patients during medical appointments.

\section{Challenges to screening for food insecurity during COVID-19}

Changes brought forth by the COVID-19 pandemic are 
important to consider in efforts to increase food insecurity screenings, because they directly impact how patients and providers communicate and interact with one another. The pandemic has made food insecurity screenings even more difficult to conduct than pre-pandemic times. Following guidance from the Centers for Disease Control and Prevention (CDC), providers can only see a restricted number of patients due to social distancing guidelines and masks are required for both providers and patients in order to limit the risk of transmitting the disease. As a result of these environmental changes, patients may not feel completely comfortable discussing sensitive topics such as food insecurity. A randomized controlled trial on the use of facemasks shows that when providers wear face coverings during consultations, patients' perceived empathy decreased (14), making it more difficult to establish and maintain a patient-provider relationship. In addition, a systematic review on the downsides of wearing face covering discusses several studies showing that healthcare workers reported difficulties communicating with patients while wearing a face covering (15).

Further, after the onset of the COVID-19 pandemic, the use of telehealth substantially increased in order to reduce the risk of infection from COVID-19 and to comply with social distancing guidelines from the CDC. Telehealth-or telemedicine-utilizes telecommunication technologies to provide health care services when patients and providers are not in the same physical location (16). In a survey of about 3,500 primary care providers and pediatricians, about $12 \%$ reported using telehealth in 2016, and over $90 \%$ of practices offered telehealth after the onset of the pandemic (17). Furthermore, the CDC analyzed data from four of the largest U.S. telehealth providers offering services in all states and found that the number of telehealth visits increased by $50 \%$ from the first quarter of 2019 to the first quarter of 2020 (18). Telehealth visits in surveillance week 13 of 2020 increased by $154 \%$ compared to the same period in 2019.

\section{Strengths and limitations of telebealth}

Telehealth has provided a crucial connection between providers and patients during an unprecedented health challenge for our nation and the world (19). The use of telehealth allowed providers to stay connected with patients and address their needs remotely (19). Some primary care physicians noted that using telehealth allowed for better access to care for some patients, as travel times to and from physician offices were eliminated (19). Additionally, delivery of care was seen as more efficient by some primary care physicians, as telehealth allows patients to be seen more quickly and frequently compared with traditional face-toface appointments (19). Some providers have noted that some benefits to communication via telehealth services 'included patients' comfort as well as providers' ability to observe patients, their home environments, and their facial expressions" (19). These aspects of telehealth make it an invaluable tool for providers to connect with patients safely and efficiently no matter the circumstance.

Despite the utility of telehealth services as a tool to connect patients and healthcare providers, telehealth comes with unique challenges to consider, many of which may make routine screenings for $\mathrm{SDOH}$, including food insecurity, difficult to achieve. For example, though telehealth services have increased access for some patients, those patients that have limited access to internet or cellular data plans required for video calls are excluded from participating in telehealth services (19). Additionally, some primary care providers note that while some medical appointments can be completed via video or telephone consultation, not all medical concerns can be addressed in this manner as physical examinations and nonverbal cues of patients are difficult to assess (19). Finally, some limitations of telehealth services as described by healthcare providers include difficulty engaging with patients and concern "about the lack of direct human contact, which made it difficult to foster the therapeutic relationship" (19). Specifically, some physicians noted that in-person appointments help to facilitate a trusting relationship between the physician and patient that is more difficult to achieve via telehealth, especially when patients are not already established with the physician and the practice (19).

Prior to the pandemic, food insecurity screenings were done in person and were largely dependent upon patients' comfort with discussing these potentially sensitive issues, which may make them feel ashamed to disclose (13). Telemedicine adds another layer of complexity because some patients may perceive video encounters to be impersonal, thus making it more difficult to establish a provider-patient relationship (20). There is some evidence that while patients find consultations done remotely via video has advantages, some patients have concerns about privacy and whether clinicians are able to perform adequate physical examination via telehealth services (21). A recent study on a sample of diabetic patients at the Veterans Affairs show that patients felt that telehealth video conferencing 
made it more difficult to establish a relationship with their providers because they felt that providers rushed conversations and paid less attention to them, leading some patients to feel they had minimal opportunity to be actively involved during appointments (20).

Creating a safe and comfortable environment for patients and providers to have vulnerable conversations may be more challenging when operating within a telehealth environment. A systematic review on patients' satisfaction with telehealth videoconferencing concludes that while patients generally felt satisfied with telehealth consultations, providers need to devote additional efforts in order to maintain the same level of communication and empathy through telehealth (22). For example, providers should listen to patients, provide them adequate time for asking questions, and invest time in building rapport with the patients (22).

Some preliminary research shows the powerful role of telemedicine can play in screening for social determinants of health and, as a result, improving healthcare utilization and health outcomes. Patient outreach via telemedicine was conducted in high-risk areas within Pittsburgh, Pennsylvania to screen patients for any difficulties with social determinants of health during the pandemic, including access to food, access to prescription medications, mental health needs, physical health needs, and financial concerns and connect patients to appropriate resources when needed (23). Researchers found patients who received the outreach services were significantly less likely to visit the emergency room than patients who did not receive outreach services. Outreach services that address these important social factors, provided through telemedicine mechanisms, has the potential to "significantly impact and improve patient barriers to care in a pandemic" (23).

In the previous example, telemedicine services were provided via telephone. However, some studies have found success and patient comfort with online screening mechanisms for food insecurity and other related social determinants of health, such as the Online Advocate $(24,25)$. For example, one qualitative study examined the perceptions of adolescent and young adult patients on an online mechanism of screening for social problems, a tool called the Online Advocate (25). This system electronically screens patients for social concerns and provides appropriate resource information for any needs identified (25). Eighty percent of patients stated they would not if the Online Advocate was included as part of their regular annual exam with their healthcare provider and $94 \%$ consented for the screening results to be shared with their healthcare provider (25).

While many patients of this study were receptive to the web-based screening tool, some did note the sensitivity of the topics could induce patient discomfort (25). As mentioned elsewhere, normalizing conversations surrounding social determinants of health within medical settings could help alleviate this discomfort and reduce stigma $(11,13)$. As telehealth becomes more integrated into medical service delivery, it will become increasingly important for practices to examine the various tools available (i.e., screening for $\mathrm{SDOH}$ via online tool $v$ s. video consultation with patients directly) and determine what mechanisms and methods are most suitable for their practice. Further, though some research has found providers perceive telehealth services to be more time efficient in some respects, additional time and consideration may be needed to ensure the patient-provider relationship is not diminished while utilizing telehealth services.

\section{Future considerations for telehealth research}

This article reviewed challenges and discussed some tools of telemedicine that have been used to address social determinants of health. No matter the screening mechanism, providers should adopt effective communication strategies to ensure that patients do not feel that telehealth consultations are less personal than in-person visits. Doing so can help reduce stigma and increase patient comfort with the discussion $(11,13)$. In addition, providers should ensure that they demonstrate empathy, which may be even more challenging given the impersonal nature of video or telephone appointments. It may be possible that screening for food insecurity with more established patients may be more effective because the patient-provider relationship has already been established. For less established patients, it may be of benefit for providers to consider screening patients in other ways, such as an electronic survey or questionnaire, as a way of introducing the information in a way that is nonthreatening for patients that are unfamiliar with providers.

Further research in this area is needed to better understand what best practices may exist in screening patients for SDOH via telehealth services. For example, researchers may consider examining patient and provider comfort discussing food insecurity via telehealth, and what factors facilitate or inhibit comfort and, subsequently, screening for food insecurity. Other areas of research might include implementation of wraparound services 
to ensure that not only do telehealth screenings occur, but that timely referrals and follow-up with patients via telehealth mechanisms also occurs with community resources. Integration of telehealth services with community organizations and resources may help ensure that patients are holistically cared for while minimizing the spread of COVID-19. Researchers and clinicians may also consider exploring different ways to potentially screen patients to determine if some are more effective than others. For example, how does screening patients via electronic questionnaire prior to telehealth appointments compare with face-to-face discussions of food insecurity via telehealth platforms or face-to-face discussions of food insecurity via traditional in-person appointments?

\section{Conclusions}

Creating processes to assess for and follow-up on food insecurity screenings is vital to ensuring at-risk patients are supported in their health. Regular screenings, provided with empathy and compassion, can help normalize conversations about food insecurity and bolster the patient-provider relationship. But screening is half the process; providers will also need to consider what to do when food insecure patients need referrals. Cross-sector collaboration between the healthcare sector and community organizations may help create a referral system that is as seamless as possible for patients (26). In the era of COVID-19 and increasing telehealth services, it is now more important than ever to screen for food insecurity, as increasing rates of food insecurity will worsen health disparities if unaddressed.

\section{Acknowledgements}

Funding: None.

\section{Footnote}

Provenance and Peer Review: This article was commissioned by the Guest Editors (Donna J. Slovensky and Donna M. Malvey) for the series "mHealth: Innovations on the Periphery" published in mHealth. The article has undergone external peer review.

Conflicts of Interest: Both authors have completed the ICMJE uniform disclosure form (available at https://dx.doi. org $/ 10.21037 / \mathrm{mhealth}-21-31)$. The series "mHealth: Innovations on the Periphery" was commissioned by the editorial office without any funding or sponsorship. The authors have no other conflicts of interest to declare.

Ethical Statement: The authors are accountable for all aspects of the work in ensuring that questions related to the accuracy or integrity of any part of the work are appropriately investigated and resolved.

Open Access Statement: This is an Open Access article distributed in accordance with the Creative Commons Attribution-NonCommercial-NoDerivs 4.0 International License (CC BY-NC-ND 4.0), which permits the noncommercial replication and distribution of the article with the strict proviso that no changes or edits are made and the original work is properly cited (including links to both the formal publication through the relevant DOI and the license). See: https://creativecommons.org/licenses/by-nc-nd/4.0/.

\section{References}

1. United States Department of Agriculture. Food Security in the U.S. Overview. 2021. Available online: https:// www.ers.usda.gov/topics/food-nutrition-assistance/foodsecurity-in-the-us/

2. Coleman-Jensen A, Rabbitt MP, Gregory CA, et al. Household food security in the United States in 2019. U.S. Department of Agriculture, Economic Service Research ERR-275. Available online: https://www.ers.usda.gov/ webdocs/publications/99282/err-275.pdf?v=1286.4

3. Feeding America. The Impact of the Coronavirus on Food Insecurity in 2020 \& 2021. 2021 Mar. Available online: https://www.feedingamerica.org/sites/ default/files/2021-03/National\%20Projections\%20 Brief_3.9.2021_0.pdf

4. Gundersen C, Ziliak JP. Food Insecurity And Health Outcomes. Health Aff (Millwood) 2015;34:1830-9.

5. Tait CA, L'Abbé MR, Smith PM, et al. The association between food insecurity and incident type 2 diabetes in Canada: A population-based cohort study. PLoS One 2018;13:e0195962.

6. Berkowitz SA, Seligman HK, Meigs JB, et al. Food insecurity, healthcare utilization, and high cost: a longitudinal cohort study. Am J Manag Care 2018;24:399-404.

7. Herman D, Afulani P, Coleman-Jensen A, et al. Food Insecurity and Cost-Related Medication Underuse Among Nonelderly Adults in a Nationally Representative Sample. Am J Public Health 2015;105:e48-59. 
8. Knight CK, Probst JC, Liese AD, et al. Household food insecurity and medication "scrimping" among US adults with diabetes. Prev Med 2016;83:41-5.

9. De Marchis EH, Torres JM, Fichtenberg C, et al. Identifying Food Insecurity in Health Care Settings: A Systematic Scoping Review of the Evidence. Fam Community Health 2019;42:20-9.

10. Messer E, Ross EM. Talking to patients about food insecurity. Nutr Clin Care 2002;5:168-81.

11. Pooler JA, Hoffman VA, Karva FJ. Primary Care Providers' Perspectives on Screening Older Adult Patients for Food Insecurity. J Aging Soc Policy 2018;30:1-23.

12. Cutts D, Cook J. Screening for Food Insecurity: ShortTerm Alleviation and Long-Term Prevention. Am J Public Health 2017;107:1699-700.

13. Palakshappa D, Doupnik S, Vasan A, et al. Suburban Families' Experience With Food Insecurity Screening in Primary Care Practices. Pediatrics 2017;140:e20170320.

14. Wong CK, Yip BH, Mercer S, et al. Effect of facemasks on empathy and relational continuity: a randomised controlled trial in primary care. BMC Fam Pract 2013;14:200.

15. Bakhit M, Krzyzaniak N, Scott AM, et al. Downsides of face masks and possible mitigation strategies: a systematic review and meta-analysis. BMJ Open 2021;11:e044364.

16. Health and Resources and Services Administration. What is telehealth? Published May 19, 2021. Available online: https://telehealth.hhs.gov/patients/understandingtelehealth/\#what-is-telehealth

17. North S. Telemedicine in the Time of COVID and Beyond. J Adolesc Health 2020;67:145-6.

18. Koonin LM, Hoots B, Tsang CA, et al. Trends in the Use of Telehealth During the Emergence of the COVID-19 Pandemic - United States, January-March 2020. MMWR

doi: $10.21037 /$ mhealth-21-31

Cite this article as: Bernhardt C, King C. Telehealth and food insecurity screenings: challenges and lessons learned. mHealth 2022;8:10.
Morb Mortal Wkly Rep 2020;69:1595-9.

19. Breton M, Sullivan EE, Deville-Stoetzel N, et al. Telehealth challenges during COVID-19 as reported by primary healthcare physicians in Quebec and Massachusetts. BMC Fam Pract 2021;22:192.

20. Gordon HS, Solanki P, Bokhour BG, et al. "I'm Not Feeling Like I'm Part of the Conversation" Patients' Perspectives on Communicating in Clinical Video Telehealth Visits. J Gen Intern Med 2020;35:1751-8.

21. Powell RE, Henstenburg JM, Cooper G, et al. Patient Perceptions of Telehealth Primary Care Video Visits. Ann Fam Med 2017;15:225-9.

22. Orlando JF, Beard M, Kumar S. Systematic review of patient and caregivers' satisfaction with telehealth videoconferencing as a mode of service delivery in managing patients' health. PLoS One 2019;14:e0221848.

23. Austin S, Pappan N, Venkat D. Improving Emergency Room Utilization During the COVID-19 Pandemic: A Telemedicine Social Determinants of Health Outreach Approach. J Community Hosp Intern Med Perspect 2021;11:485-8.

24. Fleegler EW, Lieu TA, Wise PH, et al. Families' healthrelated social problems and missed referral opportunities. Pediatrics 2007;119:e1332-41.

25. Wylie SA, Hassan A, Krull EG, et al. Assessing and referring adolescents' health-related social problems: qualitative evaluation of a novel web-based approach. J Telemed Telecare 2012;18:392-8.

26. Stenmark SH, Steiner JF, Marpadga S, et al. Lessons Learned from Implementation of the Food Insecurity Screening and Referral Program at Kaiser Permanente Colorado. Perm J 2018;22:18-093. 